\title{
Primitive divisors of sequences associated to elliptic curves
}

\author{
Matteo Verzobio
}

\begin{abstract}
Let $\{n P+Q\}_{n \geq 0}$ be a sequence of points on an elliptic curve defined over a number field $K$. In this paper, we study the denominators of the $x$-coordinates of this sequence. We prove that, if $Q$ is a torsion point of prime order, then for $n$ large enough there always exists a primitive divisor. Later on, we show the link between the study of the primitive divisors and the Lang-Trotter conjecture.
\end{abstract}

\section{Introduction}

Let $E$ be an elliptic curve defined over a number field $K, P$ a non-torsion point of $E(K)$ and $Q$ a point of $E(K)$ such that, for every $n>0, n P \neq-Q$. Since every fractional ideal of $K$ has a unique factorization, we can write

$$
(x(n P+Q))=\frac{C_{n}}{D_{n}}
$$

where $C_{n}$ and $D_{n}$ are two relatively prime integral ideals. If $K=\mathbb{Q}$, then $D_{n}$ can be represented uniquely by a positive integer. We want to understand when a term of the sequence $\left\{D_{n}\right\}_{n>0}$ has a primitive divisor, i.e., when there exists a prime ideal $\mathfrak{p}$ such that

$$
\mathfrak{p} \nmid D_{1} D_{2} \cdots D_{n-1} \text { but } \mathfrak{p} \mid D_{n} .
$$

If $\mathfrak{p}$ is a primitive divisor of $D_{n}$, then $n$ is the smallest positive integer such that

$$
n P+Q \equiv O \quad \bmod \mathfrak{p} .
$$

There are some results under the hypothesis $Q=O$. In 1988, Silverman proved the following theorem.

Theorem 1.1 (Silverman [4). If $Q=O$ and $K=\mathbb{Q}$, then $D_{n}$ has a primitive divisor for every $n$ large enough.

This result was generalized by Cheon and Hahn.

Theorem 1.2 (Cheon, Hahn [1]). If $Q=O$ and $K$ is a number field, then $D_{n}$ has a primitive divisor for every $n$ large enough. 
In the case when $Q \neq O$ it was proved, in [2], that

$$
\omega_{K}\left(\prod_{n=M}^{N+M} D_{n}\right) \gg N
$$

where $\omega_{K}$ is the function that counts the number of distinct prime divisors of an integral ideals in $K$. Thanks to this result it is reasonable to think that the result of Cheon and Hahn it is true also in the case when $Q \neq O$. We will generalize this result under the assumption that $Q$ is a torsion point of prime order.

Theorem 1.3. Let $E$ be an elliptic curve defined over a number field $K$, let $P$ be a non-torsion point and $Q$ be a torsion point of prime order. Then there exists a constant $C$, such that, if $n>C$, then $D_{n}$ has a primitive divisor.

Later on, we will show why the study of the primitive divisors is related with the elliptic analogue of Artin's conjecture, the so called Lang-Trotter conjecture.

Conjecture 1.1 (Lang-Trotter). Let $E$ be an elliptic curve defined over $\mathbb{Q}$ and $P$ be a point on $E(\mathbb{Q})$. Let

$$
N_{P}(x)=\left\{p \leq x \text { such that } p \nmid \Delta \text { and } \bar{P} \text { generates } E\left(\mathbb{F}_{p}\right)\right\},
$$

where $\bar{P}$ is the reduction of $P$ modulo $p$. Then,

$$
\# N_{P}(x) \sim \delta(P) \frac{x}{\log x}
$$

as $x$ goes to infinity.

We will study a set that it is related to $N_{P}(x)$. Let $P$ and $Q$ be two points in $E(\mathbb{Q})$ and define

$$
N_{P, Q}(x):=\left\{p \leq x \text { such that } p \nmid \Delta \text { and } \bar{Q} \in\langle\bar{P}\rangle \text { in } E\left(\mathbb{F}_{p}\right)\right\},
$$

It is clear that

$$
N_{P, Q}(x) \subseteq N_{P}(x)
$$

since if $P$ generates $E\left(\mathbb{F}_{p}\right)$, then $Q$ is in the orbit of $P$ modulo $p$.

Theorem $1.4([3])$. The set $N_{P, Q}(x)$ is infinite as $x$ goes to infinite if $P$ and $Q$ have infinite order. Furthermore, if $E$ has rank 1, then

$$
\# N_{P, Q}(x) \gg \sqrt{\log x} .
$$

We will generalize this result by showing that the condition that $E$ has rank 1 and the condition that $Q$ has infinite order are unnecessary.

Theorem 1.5. Let $E$ be an elliptic curve defined over $\mathbb{Q}$ with $P$ a non-torsion point and $Q$ in $E(\mathbb{Q})$. Then,

$$
\# N_{P, Q}(x) \gg \sqrt{\log x} .
$$


In Section 2 we introduce some basic facts on the elliptic curves that we will use in the paper. Then, in Section 3 we will prove Theorem 1.3, using the same techniques introduced by Silverman in the proof of Theorem 1.1. Finally, in Section 4. we will prove Theorem 1.5 and we will show the relation between the study of the primitive divisors of $D_{n}$ and $N_{P, Q}(x)$.

\section{Preliminaries on elliptic curves}

Let $E$ be an elliptic curve defined by the equation

$$
y^{2}+a_{1} x y+a_{3} y=x^{3}+a_{2} x^{2}+a_{4} x+a_{6},
$$

take $P$ a non-torsion point and $Q$ a torsion point. Let us define

$$
(x(n P))=\frac{A_{n}}{B_{n}}
$$

with $A_{n}$ and $B_{n}$ two relatively prime integral ideals. Given a valuation $\nu$ associated to a prime $\mathfrak{p} \in K$, define

$$
h_{\nu}(P):=\max \{0,-\nu(x(P))\}
$$

and

$$
h(P)=\frac{1}{[K: \mathbb{Q}]} \sum_{\nu \in M_{K}} n_{\nu} h_{\nu}(P),
$$

where $n_{\nu}$ is the degree of the local extension $K_{\mathfrak{p}} / \mathbb{Q}_{p}$ and $M_{K}$ is the set of all the places of $K$. Given a point $P$ in $E(K)$, we define the canonical height as in [5, Proposition VIII.9.1], i.e.

$$
\hat{h}(P)=\frac{1}{2} \lim _{N \rightarrow \infty} 4^{-N} h\left(2^{N} P\right) .
$$

First of all, we recall the properties of the height and of the canonical height that will be necessary in this paper. For details see [5, Chapter 8].

- Given a point $R$, there exists a constant $C_{R}>0$ such that, for every $S$ in $E(K)$,

$$
h(R+S) \leq C_{R}+2 h(S) .
$$

- There exists a constant $C_{E}$ such that, for every $R \in E(K)$,

$$
|h(R)-2 \hat{h}(R)| \leq C_{E} .
$$

- The canonical height is quadratic, i.e.

$$
\hat{h}(n R)=n^{2} \hat{h}(R)
$$

for every $R$ in $E(K)$. 
We will need also the following proposition.

Proposition 2.1. Given an absolute value $\nu$, for every $\varepsilon>0$ there exists an $N=N(\varepsilon, \nu, P, Q)$ such that, for every $n>N$,

$$
h_{\nu}(n P+Q) \leq \varepsilon h(n P+Q) .
$$

Proof. Define $P_{n}:=n P+Q$. Then,

$$
h\left(P_{n}\right) \geq \frac{h\left(P_{n}-Q\right)-C_{-Q}}{2} \geq \hat{h}(n P)-\frac{C_{E}}{2}-\frac{C_{-Q}}{2}=n^{2} \hat{h}(P)-\frac{C_{E}}{2}-\frac{C_{-Q}}{2}
$$

and so $h\left(P_{n}\right) \rightarrow \infty$ as $n$ goes to infinity. We conclude using Siegel's Theorem (see [5, Theorem IX.3.1]), since

$$
\lim _{h\left(P_{i}\right) \rightarrow \infty} \frac{h_{v}\left(P_{i}\right)}{h\left(P_{i}\right)}=0 .
$$

Let $S$ be the finite set of places of $K$ composed by

- all archimedean places;

- all places over primes where $E$ has bad reduction and the primes $\mathfrak{p}$ where the equation defining the elliptic curve is not minimal in $K_{\mathfrak{p}}$;

- all places over the primes ramified in $K$;

- all places over primes dividing $2 \cdot \operatorname{ord}(Q)$, where $\operatorname{ord}(Q)$ is the order of $Q$ in $E$;

- the places over the primes where $Q$ reduces to the identity.

Lemma 2.2. If $\nu$ is a place not in $S$ and $M$ is a point of $E(K)$ such that $h_{\nu}(M)>0$, then

$$
h_{\nu}(n M)=h_{\nu}(M)+2 \nu(n) .
$$

Proof. This follows from the properties of the formal group of the elliptic curves. For details see [1, Lemma page 200].

Lemma 2.3. Given $R$ and $M$ in $E(K)$, there exists a constant $C(R, M)>0$ such that

$$
\hat{h}(n R+M) \geq \hat{h}(n R)-n C(R, M),
$$

for every $n \geq 1$.

Proof. The form $\langle R, M\rangle=\hat{h}(R+M)-\hat{h}(R)-\hat{h}(M)$ is bilinear and thus

$$
\begin{aligned}
0 & =\langle n R, M\rangle-n\langle R, M\rangle \\
& =\hat{h}(n R+M)-\hat{h}(n R)-\hat{h}(M)+n(-\hat{h}(R+M)+\hat{h}(R)+\hat{h}(M)) .
\end{aligned}
$$


It follows that,

$$
\hat{h}(n R)=\hat{h}(n R+M)+n\left(-\hat{h}(R+M)+\hat{h}(R)+\hat{h}(M)-\frac{\hat{h}(M)}{n}\right)
$$

and so we can choose

$$
C(R, M)=\hat{h}(R)+\hat{h}(M) .
$$

Lemma 2.4. Let $R$ and $M$ be two points of $E, \nu$ an absolute value not in $S$ and suppose $h_{\nu}(R)>0$ and $h_{\nu}(M)>0$. Then

$$
h_{\nu}(R+M) \geq \min \left\{h_{\nu}(R), h_{\nu}(M)\right\} .
$$

Proof. We use the formal group as defined in [5]. For every $P$, define

$$
z(P):=\frac{x(P)}{y(P)}
$$

and so

$$
\operatorname{ord}_{\mathfrak{p}}(z(P))=\frac{h_{\nu}(P)}{2}
$$

where $\mathfrak{p}$ is the prime associated to $\nu$. Indeed, using the equation generating the elliptic curve and that $\nu$ is not in $S$ we have

$$
\operatorname{Bord}_{\mathfrak{p}}(x(P))=2 \operatorname{ord}_{\mathfrak{p}}(y(P))<0 .
$$

Let $j:=\min \left\{h_{\nu}(R), h_{\nu}(M)\right\}$. Therefore,

$$
z(R+M) \equiv z(R)+z(M) \equiv 0 \quad \bmod \mathfrak{p}^{\frac{j}{2}} .
$$

thanks to [5, Proposition 7.2.2.]. Thus,

$$
\frac{h_{\nu}(R+M)}{2}=\operatorname{ord}_{\mathfrak{p}}(z(R+M)) \geq \frac{j}{2}
$$

and this concludes the proof.

\section{Proof of Theorem 1.3}

We are now ready to give the proof of Theorem 1.3. We will use two trivial estimates. Let $\mu(n)$ the number of divisors of $n$. Then,

$$
\mu(n) \leq n .
$$

Given $x \in K$, define

$$
h(x):=\frac{1}{[K: \mathbb{Q}]} \sum_{\nu} n_{\nu} h_{\nu}(x)
$$

where the sum runs over all the places of $K$ and $h_{\nu}(x)=\max \{0,-\nu(x)\}$. It is well known that $h(d)=\log d$ if $d$ is in $\mathbb{Z}$ and thus

$$
\sum_{d \mid n} h(d)=\sum_{d \mid n} \log d \leq \mu(n) \log n \leq n \log n .
$$


Proof of Theorem 1.3. Suppose that $Q$ is a $r$-torsion point, with $r$ a prime. Define

$$
K:=\max _{0 \leq m \leq r-1} C_{m Q}
$$

with $C_{R}$ as defined in the previous section. Hence, for every $n$ and for every $M \in E(K)$,

$$
h(M+n Q) \leq 2 h(M)+K .
$$

Take $n>1$ and suppose that $D_{n}$ has not a primitive divisor. We will prove that this is not possible for $n$ large enough. So, for every prime $\mathfrak{q}$ over a valuation not in $S$, if $\mathfrak{q}$ divides $D_{n}$, then it divides $D_{n-k}$ for some $k>0$ and therefore

$$
k P \equiv n P+Q-(n-k) P-Q \equiv O-O \equiv O \quad \bmod \mathfrak{q} .
$$

Here $O$ is the identity of the elliptic curve and, given two point $R$ and $M$ in $E(K)$, we say that $R \equiv M \bmod \mathfrak{p}$ if the reduction of $R$ is equal to the reduction of $M$ modulo $\mathfrak{p}$. Hence,

$$
r n P \equiv r(n P+Q) \equiv r O \equiv O \quad \bmod \mathfrak{q}
$$

Let $j:=\operatorname{gcd}(r n, k)$ and by Bézout's identity there exist $a$ and $b$ such that

$$
r n a+b k=j .
$$

It follows that

$$
j P \equiv r n a P+b k P \equiv a O+b O \equiv O \bmod \mathfrak{q}
$$

and so $\mathfrak{q}$ divides $B_{j}$ for some $j<n$ that divides $r n$. If $j$ divides $n$, then

$$
Q \equiv n P+Q \equiv O \bmod \mathfrak{q}
$$

and this is absurd since $\mathfrak{q}$ is not associated to a valuation in $S$. Then, for every divisor $\mathfrak{q}$ of $D_{n}, \mathfrak{q}$ divides $B_{r n / d}$ with $d$ a divisor of $n$, coprime with $r$ and greater than $r$.

Lemma 3.1. If $\nu$ is a valuation not in $S$ associated to a prime $\mathfrak{q}$, that divide $D_{n}$ and $B_{r n / d}$ with $(r, d)=1$, then there exists a point $Q_{d}$, multiple of $Q$, such that

$$
h_{\nu}(n P+Q) \leq h_{\nu}\left(\frac{n}{d} P+Q_{d}\right)+2 h_{\nu}(d)
$$

Proof. Define

$$
P_{1}=\frac{r n}{d} P \text { and } P_{2}=n P+Q
$$

Thus,

$$
P_{1} \equiv P_{2} \equiv O \quad \bmod \mathfrak{q}
$$

and

$$
h_{\nu}\left(P_{2}\right)=h_{\nu}\left(r P_{2}\right)=h_{\nu}(r n P)=h_{\nu}\left(\frac{r n}{d} P\right)+2 h_{\nu}(d)=h_{\nu}\left(P_{1}\right)+2 h_{\nu}(d) .
$$


Since $d$ is coprime with $r$ we have $(r, d-r)=1$ and therefore

$$
a r+b(d-r)=1
$$

with $a$ and $b$ integers. Then,

$$
\begin{aligned}
\frac{n}{d} P+b Q & =a \frac{r n}{d} P+b \frac{n(d-r)}{d} P+b Q \\
& =a \frac{r n}{d} P+b\left(n P+Q-\frac{r n}{d} P\right) \\
& =(a-b) P_{1}+b P_{2} .
\end{aligned}
$$

So, thanks to Lemma 2.4

$$
h_{\nu}\left(\frac{n}{d} P+b Q\right) \geq h_{\nu}\left(P_{1}\right)=h_{\nu}\left(P_{2}\right)-2 h_{\nu}(d) .
$$

Hence, define $Q_{d}=b Q$ and this concludes the proof.

Observe that $Q_{d}$ does not depend on $\nu$, but only on $Q$ and $d$. If $\mathfrak{q}$ is the prime associated to $\nu$ and $h_{\nu}(n P+Q)>0$, then $\mathfrak{q}$ divides $D_{n}$ and so there exists $d$ such that $\mathfrak{q}$ divides $B_{n r / d},(r, d)=1$ and $d>r$. Thus, we can apply the previous lemma, obtaining that, if $h_{\nu}(n P+Q)>0$, then there exists $d$ such that

$$
h_{\nu}(n P+Q) \leq h_{\nu}\left(\frac{n}{d} P+Q_{d}\right)+2 h_{\nu}(d) .
$$

Therefore,

$$
n_{\nu} h_{\nu}(n P+Q) \leq \sum_{\substack{d \mid n \\ d>r}} n_{\nu} h_{\nu}\left(\frac{n}{d} P+Q_{d}\right)+2 n_{\nu} h_{\nu}(d)
$$

since every addend in the RHS is greater than 0 and the LHS is less than an addend in the RHS. Hence,

$$
\sum_{\nu \notin S} n_{\nu} h_{\nu}(n P+Q) \leq \sum_{\nu \notin S} \sum_{\substack{d \mid n \\ d>r}} n_{\nu} h_{\nu}\left(\frac{n}{d} P+Q_{d}\right)+2 n_{\nu} h_{\nu}(d) .
$$


So,

$$
\begin{aligned}
\frac{1}{[K: \mathbb{Q}]} \sum_{\nu \notin S} n_{\nu} h_{\nu}(n P+Q) \leq & \frac{1}{[K: \mathbb{Q}]} \sum_{\nu \notin S} \sum_{\substack{d \mid n \\
d>r}} n_{\nu} h_{\nu}\left(\frac{n}{d} P+Q_{d}\right)+2 n_{\nu} h_{\nu}(d) \\
& \leq \sum_{\substack{d \mid n \\
d>r}} h\left(\frac{n}{d} P+Q_{d}\right)+2 h(d) \\
& \leq \sum_{d \mid n} 2 h\left(\frac{n}{d} P\right)+2 h(d)+K \\
& \leq\left(\sum_{d>r} 4 \hat{h}\left(\frac{n}{d} P\right)\right)+\left(K+2 C_{E}\right) \mu(n)+2 n \log n \\
& \leq 4 \hat{h}(P) n^{2}\left(\sum_{d \mid n} \frac{1}{d^{2}}\right)+2 n \log n+\left(K+2 C_{E}\right) n \\
& \leq 4 \hat{h}(P) n^{2} \sum_{d>2} \frac{1}{d^{2}}+\left(K+2 C_{E}\right) n+2 n \log n \\
& =4 \hat{h}(P) n^{2}\left(\zeta(2)-1-\frac{1}{4}\right)+\left(K+2 C_{E}\right) n+2 n \log n \\
& \leq 1.6 n^{2} \hat{h}(P)+\left(K+2 C_{E}\right) n+2 n \log n .
\end{aligned}
$$

Now, we have to deal with the absolute values in $S$. Using Proposition 2.1 with $\varepsilon=(100 \# S)^{-1}$ we have

$$
\begin{aligned}
\frac{1}{[K: \mathbb{Q}]} \sum_{\nu \in S} n_{\nu} h_{\nu}(n P+Q) & \leq \frac{1}{[K: \mathbb{Q}]} \sum_{\nu \in S} n_{\nu} \varepsilon h(n P+Q) \\
& \leq \frac{h(n P+Q)}{100} \\
& \leq \frac{2 h(n P)+C_{Q}}{100} \\
& \leq 0.04 n^{2} \hat{h}(P)+\frac{C_{Q}+2 C_{E}}{100}
\end{aligned}
$$

for $n>\max _{\nu \in S}\{N(\nu, \varepsilon)\}$. Finally, using Lemma 2.3.

$$
\begin{aligned}
2 n^{2} \hat{h}(P) & \leq 2(\hat{h}(n P+Q)+n C(P, Q)) \\
& \leq h(n P+Q)+2 n C(P, Q)+C_{E} \\
& =\frac{\sum_{\nu \in S} n_{\nu} h_{\nu}(n P+Q)+\sum_{\nu \notin S} n_{\nu} h_{\nu}(n P+Q)}{[K: \mathbb{Q}]}+2 n C(P, Q)+C_{E} \\
& \leq 1.64 n^{2} \hat{h}(P)+J n \log n
\end{aligned}
$$

with

$$
J:=2+2 K+4 C_{E}+2 C(P, Q)
$$


and then

$$
0.36 n^{2} \leq \frac{J n \log n}{\hat{h}(P)}
$$

Taking

$$
C:=\max \left\{2,8 \frac{J^{2}}{\hat{h}(P)^{2}}, \max _{\nu \in S}\{N(\nu, \varepsilon)\}\right\}
$$

we have that, for every $n>C$, the inequality does not hold and then $D_{n}$ has a primitive divisor.

Remark 3.2. The constant $C$ is not effective since Siegel's Theorem it is not.

\section{An elliptic analogue of Artin's conjecture}

Let $E$ be an elliptic curve defined over $\mathbb{Q}$ generated by

$$
y^{2}+a_{1} x y+a_{3} y=x^{3}+a_{2} x^{2}+a_{4} x+a_{6} .
$$

Let $S_{1}$ be the finite set of primes such that $E$ is not in minimal form in $\mathbb{Q}_{p}$ and such that $E$ has bad reduction. Take $P$ a non-torsion point and $Q$ in $E(\mathbb{Q})$. We want to prove Theorem 1.5 .

Proof of Theorem 1.5. If $Q$ is in the orbit of $P$ in $E(\mathbb{Q})$, then the theorem is trivial. So, we can suppose that $n P \neq Q$ for every $n$. As defined in the introduction, let

$$
N_{P, Q}(x):=\left\{p \leq x \text { such that } p \nmid \Delta \text { and } Q \in\langle P\rangle \text { in } E\left(\mathbb{F}_{p}\right)\right\} .
$$

If $Q$ is in the orbit of $P$ modulo $p$, then there exists $n$ such that

$$
n P \equiv Q \quad \bmod p .
$$

Define

$$
x(n P+Q)=\frac{C_{n}}{D_{n}} \text { with } C_{n}, D_{n} \in \mathbb{Z} \text { and }\left(C_{n}, D_{n}\right)=1 .
$$

So, $Q$ is in the orbit of $P$ modulo $p$ if and only if $p$ divides $D_{n}$ for some $n$. If $p$ divides $D_{n}$ and it is not in $S_{1}$, so $\operatorname{ord}_{p}\left(D_{n}\right)$ is even and then

$$
\log p^{2} \leq \log \left|D_{n}\right| \leq h(n P+Q) \leq 2 h(n P)+C_{Q} \leq 4 n^{2} \hat{h}(P)+C_{Q}+2 C_{E} .
$$

Now, define

$$
N=\sqrt{\frac{2 \log x-C_{Q}-2 C_{E}}{4 \hat{h}(P)}} .
$$

Thus, if $p$ divides $D_{n}$ for $1 \leq n \leq N$, then

$$
\log p \leq 2 n^{2} \hat{h}(P)+\frac{C_{Q}}{2}+C_{E} \leq 2 N^{2} \hat{h}(P)+\frac{C_{Q}}{2}+C_{E}=\log x .
$$


So, if $p$ divides $D_{n}$ for some $1 \leq n \leq N$, then $Q$ is in the orbit of $P$ modulo $p$ and $p \leq x$. Thus, we conclude that

$$
\left\{p \mid D_{n} \text { such that } p \notin S_{1} \text { and } 1 \leq n \leq N\right\} \subseteq N_{P, Q}(x) .
$$

Thus,

$$
\omega_{\mathbb{Q}}\left(\prod_{n=1}^{N} D_{n}\right) \leq \# N_{P, Q}(x)+\# S_{1}
$$

where $\omega_{\mathbb{Q}}$ is the function that counts the number of distinct prime divisors of an integer. In [2, Theorem 1.1.], it was proved that

$$
\omega_{K}\left(\prod_{n=M}^{N+M} D_{n}\right) \gg N
$$

and then

$$
\# N_{P, Q}(x) \gg \omega_{\mathbb{Q}}\left(\prod_{n=1}^{N} D_{n}\right) \gg N \gg \sqrt{\log x}
$$

Corollary 4.1. If $P$ is a non-torsion point and $Q$ is a torsion point of prime order, then

$$
\lim _{x \rightarrow \infty} \frac{\# N_{P, Q}(x)}{\sqrt{\log x}} \geq \frac{1}{\sqrt{2 \hat{h}(P)}} .
$$

Proof. Thanks to Theorem 1.3, we know that $D_{n}$ has a primitive divisor for every $n \geq C$, with $C$ a constant depending on $E, P$ and $Q$. So,

$$
\omega_{\mathbb{Q}}\left(\prod_{n=1}^{N} D_{n}\right) \geq N-C
$$

and therefore

$$
\# N_{P, Q}(x) \geq N-C-\# S_{1}=\sqrt{\frac{\log x-C_{Q} / 2-C_{E}}{2 \hat{h}(P)}}-C-\# S_{1} .
$$

Hence,

$$
\lim _{x \rightarrow \infty} \frac{\# N_{P, Q}(x)}{\sqrt{\log x}} \geq \lim _{x \rightarrow \infty} \frac{\sqrt{\frac{\log x-C_{Q} / 2-C_{E}}{2 \hat{h}(P)}}-C-\# S_{1}}{\sqrt{\log x}}=\frac{1}{\sqrt{2 \hat{h}(P)}} .
$$




\section{References}

[1] J. Cheon and S. Hahn. The orders of the reductions of a point in the Mordell-Weil group of an elliptic curve. Acta Arith., 88(3):219-222, 1999.

[2] Graham Everest and Igor E. Shparlinski. Prime divisors of sequences associated to elliptic curves. Glasg. Math. J., 47(1):115-122, 2005.

[3] François Séguin. The two-variable Artin conjecture and elliptic analogues. PhD thesis, Queen's University, 2018.

[4] Joseph H. Silverman. Wieferich's criterion and the abc-conjecture. J. Number Theory, 30(2):226-237, 1988.

[5] Joseph H. Silverman. The arithmetic of elliptic curves, volume 106 of Graduate Texts in Mathematics. Springer, Dordrecht, second edition, 2009.

UNIVERSITÁ DI PISA, DIPARTIMENTO DI MATEMATICA, LARGO BRUNO PONTECORVO 5, PISA, ITALY

E-mail address: verzobio@student.dm.unipi.it 